\title{
Image Restoration via Phase-Derived Drift Correction of Movies of 2D Crystals Acquired with a CMOS Direct Electron Detector.
}

\author{
Agustin J. Avila-Sakar ${ }^{1}$, Blake M. Milner ${ }^{2}$, Fei Guo ${ }^{1}$ \& Wen Jiang ${ }^{1}$. \\ ${ }^{1}$ Department of Biological Sciences and \\ ${ }^{2}$ School of Biological Engineering, Purdue University, West Lafayette, Indiana.
}

Movies of graphene oxide (GO) acquired with a direct electron detector (DE-12, Direct Electron, LP, San Diego, CA), were analyzed to determine frame-to-frame spatial shifts and restore the integrated image after alignment of frames with each other. Since in a crystal most of the structural information is contained in the periodic reflections of the reciprocal lattice, we used the crystallographic phases to derive image shifts in real space. Most images were affected to a certain extent by movement, evidenced by weakening or loss of reflections in one direction or another. Shifting each frame back by the amount computed from the phase shifts and summing them up revealed the missing reflections or strengthen those that originally appeared weak.

Images were recorded on a Titan Krios electron microscope (FEI Company, Eindhoven, NL) operated at $300 \mathrm{kV}$, at a nominal magnification of $37 \mathrm{Kx}$ at the film plate level, corresponding to a pixel size of 0.91 $\AA$ on the specimen. Exposures were 1s long movies of 25 frames, each frame lasting $40.7 \mathrm{~ms}$. The electron dose was $2.95 \mathrm{e}^{-/}$pixel/frame, which corresponded to $3.5 \mathrm{e}^{-/} / \mathrm{pixel} / \AA^{2}$ on the specimen. The target defocus was $1 \mu \mathrm{m}$. Samples were held at $90^{\circ} \mathrm{K}$.

While images of GO under the conditions described above did no show any visible contrast, the 1st order reflections, $(\mathbf{1}, \overline{\mathbb{1}}, \mathbf{0}, \mathbf{0}),(\mathbf{0}, \overline{\mathbb{1}}, \mathbf{1}, \mathbf{0}),(\overline{\mathbb{1}}, \mathbf{0}, \mathbf{1}, \mathbf{0})$, of the reciprocal lattice $\left(1 / 2.13 \AA^{-1}\right)$ appeared at 0.86 Nyquist on their Fourier transforms, in the figures below labeled as 1, 2, 3, respectively. However, the full, isotropic, pattern of reflections was not always observed on images integrated over their full exposure time. More typically, one direction appeared stronger while the perpendicular one showed weaker reflections or no reflections at all, indicating some degree of movement during exposure. Inspection of individual frames revealed a common pattern of change through the exposure. The first few frames typically showed only some weak reflections or no reflections at all. The frames in the middle and end of the movie were generally of better quality, exhibiting a more isotropic pattern of reflections. To better analyze the course of change throughout the movie, select 1024x1024 image areas were integrated in groups of 5 frames at a time, which smoothened the frame-to-frame variations and revealed a gradual change in the phase of the reflections (Figure 1).

A real space drift vector was estimated by least squares fit to the phase change rates of the GO reflections. Frames in the selected interval were shifted back accordingly and added up. The applied real-space translations resulted in a restoration of the crystallographic reflection pattern in the Fourier transforms of the summed-up frames, with reflections that were first not visible appearing distinctly or weak reflections becoming stronger (figure 2). For example, the signal/background ratio of reflection 1 in that figure changed from 1.2 to 11.9 , and that of reflection 2 , changed from 5.7 to 9.8 .

One of the hallmarks of direct electron detectors, their fast acquisition rate, has enabled unprecedented recovery of data otherwise lost due to specimen or image movement during exposure [1]. This, combined with advanced online data processing and precise alignment algorithms for non-periodic 
structures, has made possible the reconstruction of small biological macromolecules to near-atomic resolution [2,3]. In our study, we show that frame-to-frame alignment for periodic specimens is also feasible by virtue of the analysis of the phase shift of crystallographic reflections.

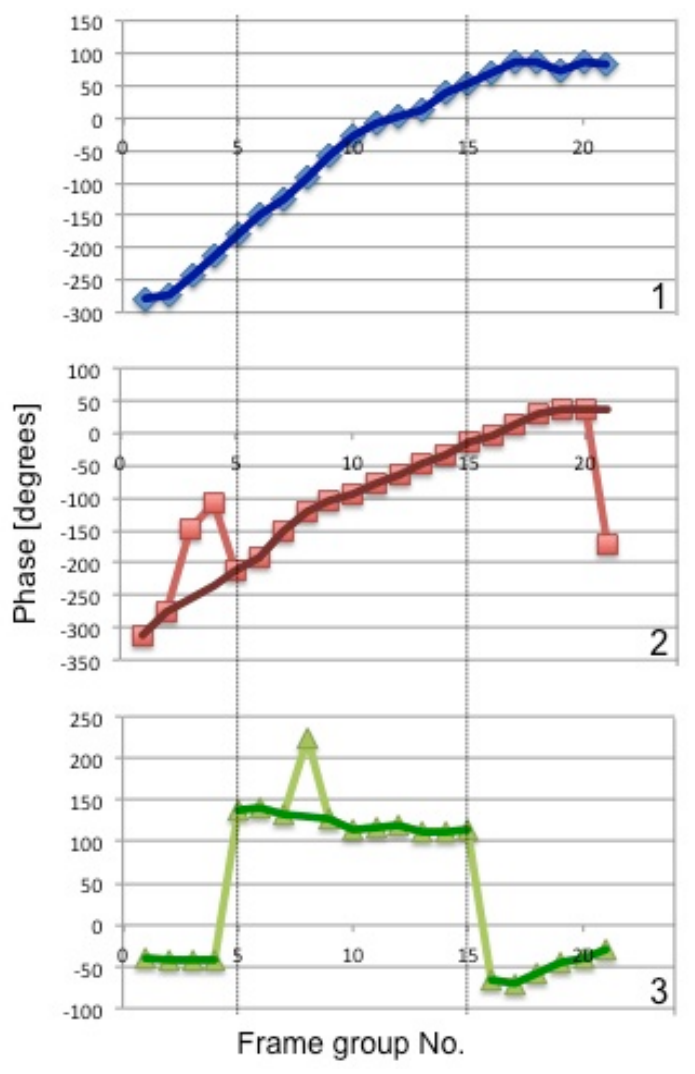

Figure 1. Time course of phase shifts of the 1st order crystallographic reflections of graphene oxide (GO) during a 1s exposure. The phases exhibited an approximately constant rate of change over the interval indicated by the vertical lines. The underlying real space drift rate, estimated by least squares fit to these phase shift rates, was $0.15 \mathrm{pixel} /$ frame, corresponding to $14 \mathrm{pm} /$ frame at the specimen.

References:

[1] Grigorieff, N., eLife 2 (2013), e00573.

[2] Li, X. et al, Nature Methods 10 (2013), p. 584.

[3] Liao, M. et al, Nature 504 (2013), p. 107.

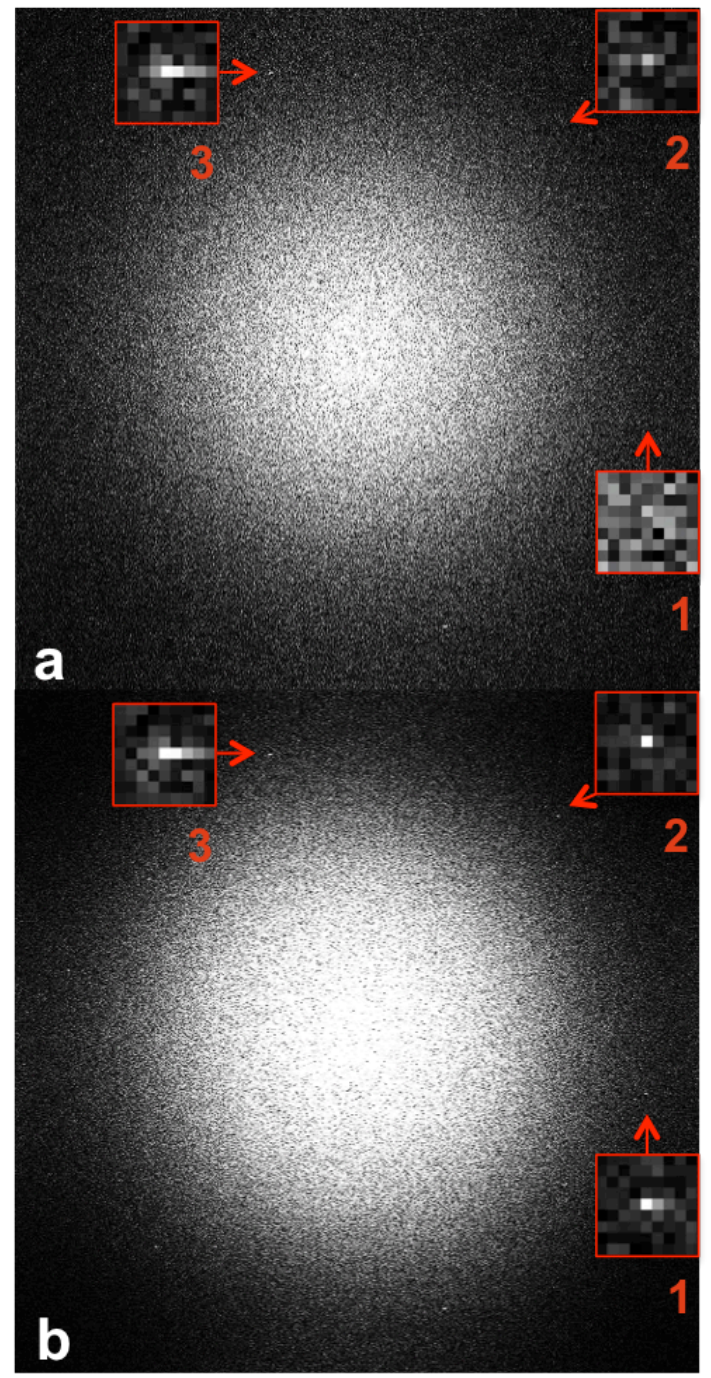

Figure 2. Fourier transforms of integrated original (a) and drift-corrected (b) images. Reflection 1, not visible in the original, became visible after correction, and reflection 2, weak in the original, grew stronger, almost doubling its signal/background ratio. 